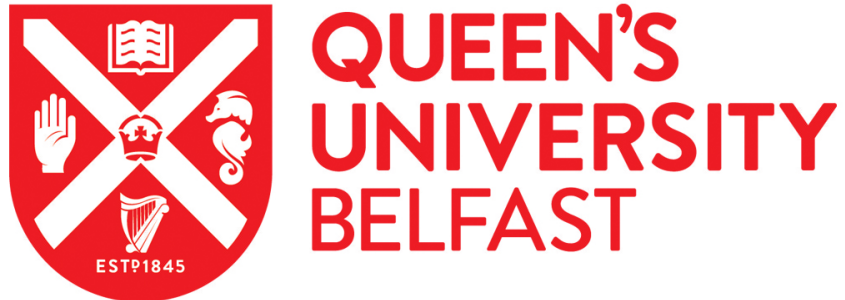

\section{The localized radial basis functions for parameterized level set based structural optimization}

-ul-Islam, S., Khan, W., Ullah, B., \& Ullah, Z. (2020). The localized radial basis functions for parameterized level set based structural optimization. Engineering Analysis with Boundary Elements, 113.

https://doi.org/10.1016/j.enganabound.2020.01.008

Published in:

Engineering Analysis with Boundary Elements

Document Version:

Peer reviewed version

Queen's University Belfast - Research Portal:

Link to publication record in Queen's University Belfast Research Portal

Publisher rights

Copyright 2020 Elsevier

This manuscript is distributed under a Creative Commons Attribution-NonCommercial-NoDerivs License

(https://creativecommons.org/licenses/by-nc-nd/4.0/), which permits distribution and reproduction for non-commercial purposes, provided the author and source are cited.

\section{General rights}

Copyright for the publications made accessible via the Queen's University Belfast Research Portal is retained by the author(s) and / or other copyright owners and it is a condition of accessing these publications that users recognise and abide by the legal requirements associated with these rights.

Take down policy

The Research Portal is Queen's institutional repository that provides access to Queen's research output. Every effort has been made to ensure that content in the Research Portal does not infringe any person's rights, or applicable UK laws. If you discover content in the Research Portal that you believe breaches copyright or violates any law, please contact openaccess@qub.ac.uk. 


\title{
The localized radial basis functions for parameterized level set based structural optimization
}

\author{
Siraj-ul-Islam ${ }^{\mathrm{a}}$,Wajid Khan ${ }^{\mathrm{a}}$, Baseer Ullah ${ }^{\mathrm{a}}$, Zahur Ullah ${ }^{\mathrm{b}}$

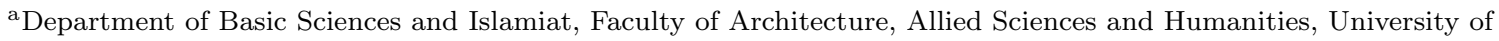 \\ Engineering and Technology, Peshawar, Pakistan. \\ b Advanced Composites Research Group, School of Mechanical and Aerospace Engineering, Queen's University Belfast, \\ Ashby Building, Belfast, BT9 5AH, UK.
}

\begin{abstract}
The current work is mainly about the implementation of the local radial basis functions (LRBFs) within the level set framework for structural optimization of two-dimensional linear elastic problems. The implicit representation of the structural geometry is accomplished through the LRBFs based level set function (LSF), and the geometry modification during the optimization process is carried out through an update of the LSF using a system of coupled ordinary differential equations (ODEs) instead of the Hamilton-Jacobi (HJ) type equation. This new implementation of LRBFs and level set method (LSM) allows essential topological changes automatically, i.e hole nucleation, hole merging with each other, and with the boundary to obtain the optimal structures. The coefficient matrix of the LRBFs is less sensitive to the values of shape parameter as well as the proposed method is mesh independent as explored during the numerical experiments performed. This new implementation is applied to distinct benchmark problems for minimum compliance with single and multiple load cases. The numerical experiments performed reveal significant insightful of the use of radial basis functions (RBFs) within the LSM framework, which may be considered as a step forward for further exploration of their intrinsic capabilities and improved performance for the solution of structural optimization problems.
\end{abstract}

Keywords: Local radial basis functions, Shape optimization, Topology optimization, Level set method, Structures, Cantilever beam.

\section{Introduction}

Topology optimization (TO) suggests the optimum material distribution for a given design domain with applied loads and boundary conditions to minimize/maximize a given objective function, e.g, compliance, mass , natural frequency, etc. TO has been emerged as an effective tool for design engineers in various industries such as aerospace, construction, automobile, etc. TO established itself as an essential part of the manufacturing industry in the design stage of micro, macro devices and nano-technologies [1].

The exiting well-known TO techniques are density based, such as homogenization and SIMP $[2-5]$, where geometry of the structure is described as a material distribution of two or three

\footnotetext{
${ }^{*}$ The author to whom all the correspondence should be addressed. Email: siraj-ul-islam@uetpeshawar.edu.pk
} 
phases. The second major class of TO techniques are based on the LSM i.e an implicit representation of the structural boundaries. Osher and Sethian [6,7] first developed the LSM for tracking the moving interfaces. Application areas of the front tracking approaches include: optics, computer animations, motion by mean curvature, fluid mechanics, image processing, solids modeling, thermal, multi-material and structural optimization problems, etc, [7-14]. The LSM based structural optimization technique was initially proposed [8], using von Mises stress based sensitivity information, which is followed by the shape sensitivity based level set approaches as reported in $[10,11]$. The use of boundary element method for LSM based structural optimization are presented in [15-17], using different objective functions respectively. The LSM with a fictitious interface energy based optimization approach has been used in [18]. A metamaterials level set based topology optimization is implemented in [19]. The maximizing diffusivity based objective function has been tailored with the LSM in [20]. The LSM with meshless element free Galerkin method is proposed for two-dimensional topology optimization problems for minimum compliance in $[21,22]$. The level set based method using shape sensitivity analysis for the optimisation of an inhomogeneous drum for the frequency response was proposed in [23]. The latest trends and developments in the field of LSM based structural optimization can be found in the review papers $[24,25]$. Detailed discussion of previous research work relevant to the proposed approach is given below.

The LSM uses the HJ equation for the evolution of LSF has the tendency of producing large gradients near the boundaries. The undesirable oscillations often destabilizes the method in the absence of any controlling mechanism of the wild gradients [25]. The poor accuracy of the LSF ultimately leads to poor approximation of the boundary normal. One option to maintain accuracy of the gradient is the re-initialization of the LSF to a signed distance function after some fixed number of iterations, during the evolution process [25], where the zero level set (iso-countour) is approximated using the finite element method (FEM) shape functions and then the discritized iso-countour distances are calculated $[26,27]$. The second technique is based on the solution of either particularly tailored partial differential equation $[11,28]$ or the solution of a boundary value problem with fast marching method [7].

Though, the LSM has the ability to effectively handle the complex topological changes during the evolution process, but still the final optimal solution is seriously dependent on the initial geometry of the structure. This dependence is due to the fact that the conventional LSM cannot nucleate holes during the evolution for two-dimensional structural optimization problems. The pre-existing holes can only cancel or merge with each other during the evolution towards an optimal structure. To handle this issue, several efforts have been made. One option is to use the topological derivatives [29], which can be implemented using two different approaches. Firstly, holes can be inserted in a region where the topological derivative has small values [30,31]. Secondly, using the modified HJ equation with topological sensitivity information for hole nucleation $[22,32,33]$. However, despite of some advantages of these approaches, the difficulty of switching among the shape and topological derivatives and controlling surface functions [31,34,35] have often been found inconvenient.

In the last few decades, the RBFs $[36,37]$ have gained much attention for the solution of structural optimization problems within the LSM framework. In this regrad an initial implementation can be found in the work of Wang and Wang [35], which was further explored in details by Wang et.al [38] for improved efficiency and performance. In the RBFs implementation the natural velocity extension allows the hole nucleation without the need of topological derivatives, alleviating the dependency of the optimal design on the initial guessed design.

There are variants of the RBFs in terms of global, local and compactly supported domain. 
The global radial basis functions (GRBFs) based LSM (GRBFs-LSM) has been introduced in [35] for structural topology optimization, where the original HJ equation is transformed into a set of ODEs following the method of lines. The contributions reported in [39-41] have pinpointed a new field of application for the RBFs (in case of globally and compactly supported RBFs) in the structural optimization.

Similar to LRBFs $[42,43]$ there is another type of radial basis functions which are compactly supported that is CS-RBFs. In case of CS-RBFs the coefficient matrix is sparse and strictly positive definite. The CS-RBFs based LSM (CS-RBFs-LSM) has been applied to structural shape and topology optimization and compliant mechanisms problems [44-47]. The CS-RBFs strongly depend on appropriate support radius. The right selection of support radius and smoothness of the function require the user experience $[36,48]$. The CS-RBFs are not suitable for data points which are further apart from the support size, also if the data points are far closer to each other, in this case the benefit of compact support may be lost [36]. Also the CS-RBFs has relatively slow rate of convergence and it is difficult to get high convergence for large scale interpolation problems $[49,50]$.

In order to explore the associated advantages, shortcomings in terms of convergence, stability, shape parameter sensitivity, mesh dependency and computational efficiency, a detailed study is carried out in this paper for the use of LRBFs and its comparison with GRBFs and CS-RBFs. To the authors knowledge this study has never been reported in the literature of structural optimization. Therefore, the main aim of the present work can be considered as a step forward to further enhance the utilization of RBFs for the solution of shape and topology optimization problems. In the last few decades, the localized RBFs appeared in the literature in variety of real world applications like computational geosciences [51], heterogeneous media [52], metal casting [53], turbulent flows [54], option pricing [55], solid mechanics and elastic plastic deformation [56] and many other [57].

Initially, we formulated the LRBFs based LSM (LRBFs-LSM) for the numerical solution of minimum compliance of two-dimensional structural optimization problems. The strain energy density field is obtained by the finite element method (FEM) using ersatz material technique [11]. The structural geometry is implicitly presented with the LRBFs. The conventional HJ equation is transformed to a system of ODEs. An approximate re-initialization is used $[41,58]$ during the evolution process. Due to the local support-domain, the LRBFs-LSM requires less memory storage as compared to the GRBFs-LSM, which has a full dense matrix representation. In addition, the LRBFs-LSM is an efficient localised version of the GRBFs-LSM, which avoids breakdown during propagation and unstable solution [41]. Since the system matrix of the GRBFsLSM is notoriously ill-conditioned due to its strong dependence on the shape parameter value, therefore, LRBFs, which produces a well-conditioned system matrix of the size of the local subdomain, within the LSM framework. In addition the numerical tests performed suggests that due to one time inversion of a small collocation matrix, the LRBFs based collocation method is computationally more efficient. It has been also observed that the final solution obtained through LRBFs-LSM is comparatively less mesh and shape parameter dependent, which is an important aspect of the implementation of LRBFs in the present work. The numerical test problems of various shapes, with single and multiple loads, related to the field of topology optimization are chosen to describe the successful implementation of the proposed method in terms of accuracy, convergence and insensitivity of the final structure to the initial design.

The rest of the paper is organised as follows. The LSM is presented in Section 2. Some details about he GRBFs and the LRBFs are presented in Sections 3. In Section 4, the compliance minimization problem is described. Numerical results are described in Section 5. In section 6, 
some conclusion are drawn.

\section{Conventional Level set method based optimization}

The conventional LSM $[10,11]$ has been used to track evolution of shapes towards optimum structures. The implicit representation of LSF is $\Gamma=\left\{\mathbf{x} \in \Omega \subset R^{d} \mid \Psi(\mathbf{x})=0\right\}$, which represents the initial interface. Let $D \subset R^{d}$ be a closed set which include all admissible shapes i.e. $\Omega \subset D$, following [59], mathematically the function $\Psi$ holds as:

$$
\Psi(\mathbf{x}) \begin{cases}<0 & \text { if } \mathbf{x} \in \Omega \\ =0 & \text { if } \mathbf{x} \in \Gamma \\ >0 & \text { if } \mathbf{x} \notin \Omega .\end{cases}
$$

Evolution of the LSF is performed through the following HJ equation [60]

$$
\frac{\partial \Psi}{\partial t}+v|\nabla \Psi|=0, \quad \Psi(\mathbf{x}, t=0)=\Psi_{0}(\mathbf{x}),
$$

where $v$ shows the movement in normal direction and $t$ is the pseudo time.

In the conventional LSM, Eq. (1) is used to model the moving front in the Eulerian sense. The upwind scheme is used to solve Eq. (2) on a cartesian grid [11] with the condition that the time stepping must satisfy the Courant-Friedrichs-Lewy (CFL) condition. During the evolution, the LSF often becomes very flat or peaked, which causes numerical instabilities. Hence, a reinitialization scheme is often required periodically to maintain the signed distance property of the LSF for controlling the unwanted peaks. The conventional LSM based structural optimization techniques are strongly dependent on the initial guess designs due to their inability of hole nucleation for $2 D$ problems as discussed in $[11,38]$.

\section{Radial basis functions within the LSM framework}

A univariate radial basis functions centered at $\mathbf{x}_{i}$ is defined as [61]

$$
\Psi_{i}(\mathbf{x})=\psi\left(\left\|\mathbf{x}-\mathbf{x}_{i}\right\|\right)
$$

where $\|$.$\| is some norm like the Euclidean norm on the R^{2}$ and $\mathbf{x}=(x, y)$. The multiquadric (MQ) radial basis function is given by

$$
\Psi_{i}(\mathbf{x})=\sqrt{\left(\mathbf{x}-\mathbf{x}_{i}\right)^{2}+c^{2}},
$$

where the constant $c$ is the shape parameter, which determines the steepness and flatness of the RBF. There is a large family of radial basis functions, which include: multiquadric, inverse multiquadric, Gaussian, compactly supported and thin-plate spline, etc. In Table 1 different type of radial basis functions are given. Among these functions the MQ and thin-plate splines are the best ones for scattered data interpolation as concluded by Franke [62]. 
Table 1: Different types of RBFs.

\begin{tabular}{lr}
\hline Name & $\Psi(x)$ \\
\hline Multiquadric & $\sqrt{\left(r^{2}+c^{2}\right)}$ \\
Inverse multiquadric & $\frac{1}{\sqrt{\left(r^{2}+c^{2}\right)}}$ \\
Gaussian & $e^{-c r}$ \\
Compactly supported & $(1-r)^{m}{ }_{+} P(r)$ \\
Thin-plate spline & $r^{2} \ln (r)$ \\
\hline
\end{tabular}

\subsection{Global radial basis functions}

To overcome some of the shortcomings of the conventional LSM, an implicit modelling technique based on the GRBFs was proposed in [35], which affectively achieved automatic handling of topological changes as well as smoothness of the structural boundary. The GRBFs and LSM based structural optimiztion can be found in $[35,38,58]$. Here, we briefly review the GRBFs implementation with in the LSM framework. The MQ RBFs interpolation with $N$ nodes is defined as

$$
\Psi(\mathbf{x})=\sum_{i=1}^{N} \alpha_{i} \psi_{i}(\mathbf{x})+P(\mathbf{x}),
$$

where $\alpha_{i}$ are the unknown coefficients of the MQ RBFs and $P(\mathbf{x})=\beta_{0}+\beta_{1} x+\beta_{2} y$ is a linear polynomial.

Using the orthogonality condition and function values at $N$ knots. The following system of $N+3$ equations is obtained:

$$
\mathbf{H} \alpha=\mathbf{f},
$$

where

$$
\begin{aligned}
& \mathbf{H}=\left[\begin{array}{cc}
A & P \\
P^{\prime} & 0
\end{array}\right], \\
& \mathbf{A}=\left[\begin{array}{ccc}
\psi_{1}\left(\mathbf{x}_{1}\right) & \ldots & \psi_{N}\left(\mathbf{x}_{1}\right) \\
\ldots & \ddots & \ldots \\
\psi_{1}\left(\mathbf{x}_{N}\right) & \ldots & \psi_{N}\left(\mathbf{x}_{N}\right)
\end{array}\right] \\
& \mathbf{P}=\left[\begin{array}{ccc}
1 & x_{1} & y_{1} \\
\vdots & \vdots & \vdots \\
1 & x_{N} & y_{N}
\end{array}\right], \\
& \boldsymbol{\alpha}=\left[\begin{array}{llllll}
\alpha_{1} & \ldots & \alpha_{N} & \beta_{0} & \beta_{1} & \beta_{2}
\end{array}\right]^{T} \text {, }
\end{aligned}
$$

and

$$
\mathbf{f}=\left[\begin{array}{llllll}
f_{1} & \ldots & f_{N} & 0 & 0 & 0
\end{array}\right]^{T} .
$$

Equation (5) becomes as

$$
\Psi=\psi^{T} \alpha,
$$


where $\Psi^{T}=\left[\begin{array}{lllll}\psi_{1} \ldots \psi_{N} & 1 & x & y\end{array}\right]$.

Introducing the pseudo-time in the coefficients $\alpha$, we have

$$
\Psi(\mathbf{x})=\psi(\mathbf{x})^{T} \alpha(t)
$$

Putting the value of $\Psi(\mathbf{x})$ in Eq. (2), the resulting ODEs are as follows

$$
\mathbf{H} \frac{d \alpha}{d t}+\mathbf{B}(\alpha, t)=0
$$

where

$$
\mathbf{B}(\alpha, t)=\left[\begin{array}{c}
v\left(\mathbf{x}_{1}\right)\left|\nabla \Psi\left(\mathbf{x}_{1}\right)^{T} \alpha\right| \\
\vdots \\
v\left(\mathbf{x}_{\mathbf{N}}\right)\left|\nabla \Psi\left(\mathbf{x}_{N}\right)^{T} \alpha\right| \\
0 \\
0 \\
0
\end{array}\right] .
$$

Using the first-order forward Euler's method for the solution of coupled non-linear ODEs, the approximate solution is given by:

$$
\alpha\left(t_{i+1}\right)=\alpha\left(t_{i}\right)+d t H^{-1} \mathbf{B}\left(\alpha\left(t_{i}\right), t_{i}\right),
$$

where $d t$ is the time step size and $t_{i}$ is the $i$-th time step.

\subsection{Local radial basis functions}

In the present study, a local form of the RBFs is investigated in details within the LSM framework for its usefulness and improved efficiency. The local radial basis function interpolant is defined as [63]:

$$
\Psi_{n}(\mathbf{x})=\sum_{k \in I_{i}} \lambda_{i} \psi_{i}\left(\left\|\mathbf{x}-\mathbf{x}_{\mathbf{i}}\right\|\right)
$$

where $I_{i}$ is a vector associated with $\mathbf{x}_{i}$ which contains the center number and indices of $n-1$ neighboring centers. Every center and its $n-1$ neighboring points is called a stencil. Similar to the LRBfs, the CS-RBFs also produces the sparse matrix due to the local support domain. The CS-RBFs based interpolation can obtained from Eq.(11) if we replace the MQ RBF with the well known compactly supported function. The local support domain with 5 points stencil is depicted in Fig. 1. The expansion coefficients $\lambda_{i}$ can be obtained in similar way as in GRBFs using the interpolation condition, but the interpolation is performed on a local support domain instead of global support domain.

In the localised version of the RBFs approximation, the $m$-th order derivative of $\Psi(\mathbf{x})$ at $\left(x_{i}, y_{i}\right)$ is computed with respect to $\mathbf{x}$ with the help of neighbouring nodes $\left\{\left(x_{i_{1}}, y_{i_{1}}\right),\left(x_{i_{2}}, y_{i_{2}}\right),\left(x_{i_{3}}, y_{i_{3}}\right), \ldots,\left(x_{i_{n_{i}}}, y_{i_{n_{i}}}\right)\right\} \subset\left\{\left(x_{1}, y_{1}\right),\left(x_{2}, y_{2}\right),\left(x_{3}, y_{3}\right), \ldots,\left(x_{N^{2}}, y_{N^{2}}\right)\right\}$ as:

$$
\Psi_{x}^{(m)}\left(x_{i}, y_{i}\right) \approx \sum_{k=1}^{n_{i}} \lambda_{i_{k}}^{(m)} \psi\left(x_{i_{k}}, y_{i_{k}}\right), \quad i=1,2, \ldots \ldots N^{2}
$$

where $n_{i} \ll N$. The coefficients $\lambda_{i_{k}}^{(m)}$ are obtained by putting value of the MQ RBF into Eq. (12) as:

$$
\Psi_{x}^{(m)}\left(\left\|x_{i}-x_{l}\right\|,\left\|y_{i}-y_{l}\right\|\right)=\sum_{k=1}^{n_{i}} \lambda_{i_{k}}^{(m)} \psi\left(\left\|x_{i_{k}}-x_{l}\right\|,\left\|y_{i_{k}}-y_{l}\right\|\right), \quad l=i_{1}, i_{2}, i_{3} \ldots i_{n_{i}} .
$$




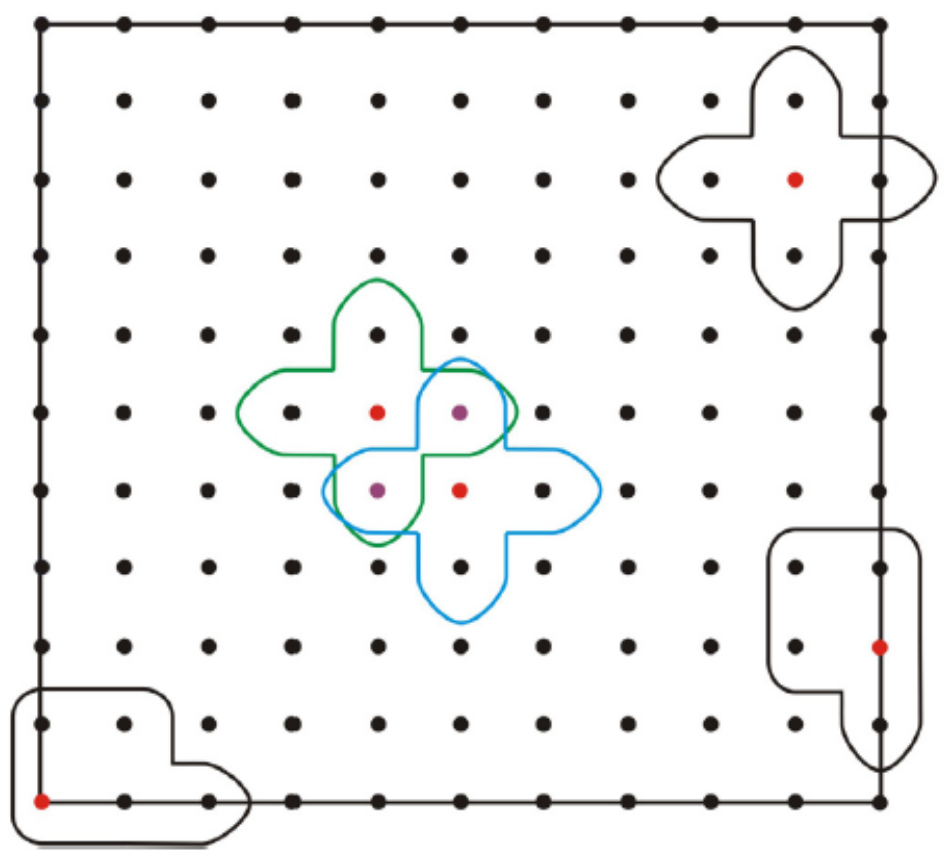

Figure 1: Schematic diagram of 5 points stencil.

Equation (13) can be written as:

$$
\left[\begin{array}{c}
\Psi_{i_{1}}^{(m)}\left(x_{i}, y_{i}\right) \\
\Psi_{i_{2}}^{(m)}\left(x_{i}, y_{i}\right) \\
\vdots \\
\Psi_{i_{n_{i}}}^{(m)}\left(x_{i}, y_{i}\right)
\end{array}\right]=\left[\begin{array}{cccc}
\psi_{i_{1}}\left(x_{i_{1}}, y_{i_{1}}\right) & \psi_{i_{2}}\left(x_{i_{1}}, y_{i_{1}}\right) & \cdots & \psi_{i_{n_{i}}}\left(x_{i_{1}}, y_{i_{1}}\right) \\
\psi_{i_{1}}\left(x_{i_{2}}, y_{i_{2}}\right) & \psi_{i_{2}}\left(x_{i_{2}}, y_{i_{2}}\right) & \cdots & \psi_{i_{n_{i}}}\left(x_{i_{2}}, y_{i_{2}}\right) \\
\vdots & \vdots & \ddots & \vdots \\
\psi_{i_{1}}\left(x_{i_{n_{i}}}, y_{i_{n_{i}}}\right) & \psi_{i_{2}}\left(x_{i_{n_{i}}}, y_{i_{n_{i}}}\right) & \cdots & \psi_{i_{n_{i}}}\left(x_{i_{n_{i}}}, y_{i_{n_{i}}}\right)
\end{array}\right]\left[\begin{array}{c}
\lambda_{i_{1}}^{(m)} \\
\lambda_{i_{2}}^{(m)} \\
\vdots \\
\lambda_{i_{n_{i}}}^{(m)}
\end{array}\right]
$$

where

$$
\psi_{l}\left(x_{k}, y_{k}\right)=\psi_{x}\left(\left\|x_{k}-x_{l}\right\|,\left\|y_{k}-y_{l}\right\|\right), \quad l=i_{1}, i_{2}, i_{3}, \ldots i_{n_{i}},
$$

for each $k=i_{1}, i_{2}, i_{3}, \ldots . i_{n_{i}}$.

In matrix notational representation, the above equation can be written as:

$$
\boldsymbol{\psi}_{n_{i}}^{(m)}=\mathbf{A}_{n_{i}} \boldsymbol{\lambda}_{n_{i}}^{(m)}
$$

where

$$
\begin{gathered}
\Psi_{n_{i}}^{(m)}=\left[\begin{array}{llll}
\psi_{i_{1}}^{(m)}\left(x_{i}, y_{i}\right) & \psi_{i_{2}}^{(m)}\left(x_{i}, y_{i}\right) & \cdots & \psi_{i_{n_{i}}}^{(m)}\left(x_{i}, y_{i}\right)
\end{array}\right]^{T}, \\
A_{n_{i}}=\left[\begin{array}{cccc}
\psi_{i_{1}}\left(x_{i_{1}}, y_{i_{1}}\right) & \psi_{i_{2}}\left(x_{i_{1}}, y_{i_{1}}\right) & \cdots & \psi_{i_{n_{i}}}\left(x_{i_{1}}, y_{i_{1}}\right) \\
\psi_{i_{1}}\left(x_{i_{2}}, y_{i_{2}}\right) & \psi_{i_{2}}\left(x_{i_{2}}, y_{i_{2}}\right) & \cdots & \psi_{i_{n_{i}}}\left(x_{i_{2}}, y_{i_{2}}\right) \\
\vdots & \vdots & \ddots & \vdots \\
\psi_{i_{1}}\left(x_{i_{n_{i}}}, y_{i_{n_{i}}}\right) & \psi_{i_{2}}\left(x_{i_{n_{i}}}, y_{i_{n_{i}}}\right) & \cdots & \psi_{i_{n_{i}}}\left(x_{i_{n_{i}}}, y_{i_{n_{i}}}\right)
\end{array}\right], \\
\lambda_{n_{i}}^{(m)}=\left[\begin{array}{llll}
\lambda_{i_{1}}^{(m)} & \lambda_{i_{2}}^{(m)} & \cdots & \lambda_{i_{n_{i}}}^{(m)}
\end{array}\right]^{T} .
\end{gathered}
$$

Consequently, we obtain,

$$
\boldsymbol{\lambda}_{n_{i}}^{(m)}=\mathbf{A}_{n_{i}}^{-1} \boldsymbol{\psi}_{n_{i}}^{(m)}
$$


Using Eq. (14) in Eq. (12) we get,

$$
\boldsymbol{\Psi}_{x}^{(m)}\left(x_{i}, y_{i}\right)=\left(\boldsymbol{\lambda}_{n_{i}}^{(m)}\right)^{T} \boldsymbol{\psi}_{n_{i}}
$$

where

$$
\boldsymbol{\psi}_{n_{i}}=\left[\begin{array}{llll}
\psi\left(x_{i_{1}}, y_{i_{1}}\right) & \psi\left(x_{i_{2}}, y_{i_{2}}\right) & \cdots & \psi\left(x_{i_{n_{i}}}, y_{i_{n_{i}}}\right)
\end{array}\right]^{T} .
$$

Similarly, the derivative approximation w.r,t. $y$ is given as:

$$
\Psi_{y}^{(m)}\left(x_{i}, y_{i}\right) \approx \sum_{k=1}^{n_{i}} \mu_{i_{k}}^{(m)} \psi\left(x_{i_{k}}, y_{i_{k}}\right), \quad i=1,2, \ldots \ldots N^{2} .
$$

The corresponding coefficients $\mu_{i_{k}}^{(m)}$ can be found as follow:

$$
\boldsymbol{\mu}_{n_{i}}^{(m)}=\mathbf{A}_{n_{i}}^{-1} \boldsymbol{\psi}_{n_{i}}^{(m)}
$$

The derivatives approximation obtained above leads to the approximation of PDE (2) as

$$
\begin{gathered}
\frac{\partial \Psi(x, y)}{\partial x}+\frac{\partial \Psi(x, y)}{\partial y}=\left(\lambda_{n_{i}}^{(1)}+\mu_{n_{i}}^{(1)}\right)^{T} \Psi_{n_{i}}, \\
\Psi_{n_{i}}=\left[\begin{array}{llll}
\psi\left(x_{i_{1}}, y_{i_{1}}\right) & \psi\left(x_{i_{2}}, y_{i_{2}}\right) & \cdots & \psi\left(x_{i_{n}}, y_{i_{n}}\right)
\end{array}\right]^{T} .
\end{gathered}
$$

By substituting the LRBFs interpolating function and Eq. (18) into the PDE (2) and collocating at the nodes $\mathbf{x}_{i}$, we obtain

$$
\frac{d \Psi}{d t}+v\left(\left(\lambda_{n_{i}}^{(1)}+\mu_{n_{i}}^{(1)}\right)^{T} \Psi_{n_{i}}\right)=0 .
$$

Eq.(19) is the system of non-linear ODEs and its numerical solution can be obtained by several well-known ODEs solver such as Euler's method, Runge-Kutta, Adam Bashforth and Adams Moulton methods. In this study, we have used the first order forward Euler's method due to its simplicity and implementation in practical problems. Using the first-order forward Euler's method for the solution of coupled non-linear ODEs, the approximate solution is given by:

$$
\mathbf{\Psi}\left(t_{i+1}\right)=\mathbf{\Psi}\left(t_{i}\right)+d t \mathbf{B}\left(\mathbf{\Psi}\left(t_{i}\right), t_{i}\right),
$$

where $d t$ is the time step size and $t_{i}$ is the $i$-th time step.

During the numerical implementation, Eq.(20) may suffer from numerical instability. The RBFs based level set method cause undesired solution in evolutionary models. To tackle this problem, [41] proposed the normalization procedure and elimination of the gradient part from the matrix B by setting $|\nabla \Psi|=1$, Hence following [41] the approximate re-initialization procedure can be written as

$$
\Psi^{\prime \prime}=\frac{\Psi}{\operatorname{mean}\left(\left|\nabla \Psi_{1}^{0}\right|,\left|\nabla \Psi_{2}^{0}\right|, \ldots\left|\nabla \Psi_{r}^{0}\right|\right)} .
$$

To prevent an unbounded growth of the LSF, an approximate $\delta(\Psi)$ is used, which is given by

$$
\delta(\Psi)= \begin{cases}0, & \Psi>\Delta, \\ \frac{3}{4 \Delta}\left(1-\frac{\Psi^{2}}{\Delta^{2}}\right), & -\Delta \leq \Psi \leq \Delta, \\ 0, & \Psi<-\Delta,\end{cases}
$$

where $\Delta$ is used as a threshold to bound the value of LSF. Thus the final updated scheme for the $\Psi$ is

$$
\Psi\left(t_{i+1}\right)=\Psi^{\prime \prime}\left(t_{i}\right)+d t \hat{B}\left(\Psi^{\prime \prime}\left(t_{i}\right), t_{i}\right)
$$


where $\hat{B}$ is given by

$$
\hat{B}=\left[\begin{array}{c}
v\left(x_{1}, t_{i}\right) \delta\left(\Psi\left(x_{1},\left(t_{i}\right)\right)\right) \\
\vdots \\
v\left(x_{N}, t_{i}\right) \delta\left(\Psi\left(x_{N},\left(t_{i}\right)\right)\right) \\
0 \\
0 \\
0
\end{array}\right] .
$$

\section{Compliance minimization problem}

In the present study, the LRBFs-LSM based approach has been applied for the solution of classical shape and topology optimization problems for minimum compliance under a volume constrained. The compliance minimization formulation for linear elastic structure with the static loading under the volume constraint is given as

$$
\text { Minimize: } \quad J(\boldsymbol{u}, \Psi)=\int_{\Omega} \epsilon(\boldsymbol{u}): C: \epsilon(\boldsymbol{u}) H(\Psi) d \Omega,
$$

subject to the constraints:

$$
\left\{\begin{array}{l}
a(\boldsymbol{u}, \delta \boldsymbol{u}, \Psi)=l(\delta \boldsymbol{u}, \Psi), \quad \forall \quad \delta \boldsymbol{u} \in H^{1} \\
G(\Psi)=\int_{\Omega} H(\Psi) d \Omega-V_{r e q} \leq 0,\left.\quad u\right|_{\Gamma_{u}}=\bar{u} \\
C: \epsilon(\boldsymbol{u}) . n=\hat{t}, \quad \text { on } \quad \Gamma_{t} \\
\boldsymbol{u}=\overline{\boldsymbol{u}}, \quad \text { on } \quad \Gamma_{u},
\end{array}\right.
$$

where $a(\boldsymbol{u}, \delta \boldsymbol{u}, \Psi)$ and $l(\delta \boldsymbol{u}, \Psi)$ in Eq. (24) are given as

$$
a(\boldsymbol{u}, \delta \boldsymbol{u}, \Psi)=\int_{\Omega} \epsilon(\boldsymbol{u}): C: \epsilon(\boldsymbol{u}) H(\Psi) d \Omega
$$

and

$$
l(\delta \boldsymbol{u}, \Psi)=\int_{\Gamma_{t}} \hat{t} \delta \boldsymbol{u} d \Gamma+\int_{\Omega} b \delta \boldsymbol{u} H(\Psi) d \Omega .
$$

In Eq. (23) $J(\Psi)$ is the objective function, $\boldsymbol{u}$ is the displacement, $\epsilon$ is the linearized strain tensor, $C$ the Hooke's elasticity tensor and $H$ is the Heaviside function. In Eq. (24), $G(\Psi)$ is the constraint introduced to limit the material usage and $V_{r e q}$ is the target volume. The specific displacement $\bar{u}$ is given on Dirichlet boundary $\Gamma_{u}$ and $\hat{t}$ is the prescribed traction on traction boundary $\Gamma_{t}$.

In the literature of LSM based structural optimization the concept of shape derivatives has been used for determining the associated sensitivities (for details see $[10,11]$ ). In case of minimum compliance problem, the normal velocity $v_{N}$ along the free boundary is defined as

$$
v_{N}=\epsilon(\boldsymbol{u}) C \epsilon(\boldsymbol{u})-\lambda .
$$

In Eq. (27) $\lambda$ is the Lagrange multiplier to handle the volume constraint and is updated by the following scheme [58]

$$
\lambda^{k+1}=\left\{\begin{array}{lc}
\mu G^{k} & k \leq N_{i t} \\
\lambda^{k}+\gamma^{k} G & k>N_{i t},
\end{array}\right.
$$


where $\gamma$ and $\mu$ are the optimization parameters and $k$ is the time loop iteration. The number $N_{i t}$ denotes the number of iterations to relax the volume constraints. The parameter $\gamma$ is updated by the following formula

$$
\gamma^{k+1}=\operatorname{Min}\left(\gamma^{k}+d \gamma, \gamma_{\max }\right), \quad k>N_{i t} .
$$

The solution of the optimization problem is carried out as per following algorithm:

1. Initialize the LSF as a signed distance function.

2. Initialize radial basis function using Eq. (11)

3. Solve the linear elasticity problem through FEM and the Ersatz material approach.

4. Calculate shape sensitivities and normal velocities accordingly.

5. Solve the level set equation Eq. (21) to update the LSF.

6 . Check the termination condition, if satisfied, stop, otherwise repeat steps $3-6$.

\section{Numerical experiments and discussions}

In this section, the proposed method is applied to some bench-mark problems of minimum compliance. In all the test cases, material properties are chosen as: Poisson's ratio $v=0.3$, Young's modulus $E=1$, for solid material and $E=10^{-09}$ for void material. The optimization parameters $\mu, \gamma, \gamma_{\max }, \Delta$ and $d t$ are assigned the values of $2,0.05,0.05,10$ and 0.5 , respectively. The value of $N_{i t}$ is 20 for all test problems unless otherwise specified. A $100 \mathrm{~N}$ load is used for all the test problems. The optimization process stops once the relative errors of the objective function values is less than $10^{-03}$ in consecutive 15 iterations and the relative errors between the consecutive volume fraction is less than $10^{-03}$. All the investigated examples are implemented using Matlab on the Intel $^{R}$ Cor $^{i 7} \mathrm{hp}$ laptop with processor 2.50 to $2.60 \mathrm{GHz}$ and RAM $8 \mathrm{~GB}$. A local support domain of size 14 with a fixed mesh of four nodes and bilinear elements are used.

Test Problem 1. Consider the cantilever beam test case with dimension $2 \times 1$. The left boundary is constrained and the middle point of right boundary is subjected to a load in the vertical direction, as illustrated in Fig. 2a. The required volume fraction is $V_{r e q}=0.5 V_{0}$. A mesh of size $60 \times 30=1800$ is used to discretize the problem domain, and the value of $c$ is 2 . The value of $N_{i t}$ is 30 .

The automatic hole insertion, expansion and merging can be observed throughout the optimization process untill the required volume fraction has been achieved. The final optimal topology is obtained after 96 iterations as shown in Fig. 2f, which confirms the similarity with the final optimal topologies for this benchmark test problem in the literature [11,38,64,65]. The hole nucleation at appropriate locations is prominent during the optimization process. The proposed method handles the topological changes effectively during the optimization process.

We validate the LRBFs-LSM method with the GRBFs-LSM presented in [58] for the same problem using the same mesh size and same parameters except, $c=10^{-01}$. We have implemented the GRBFs-LSM and CS-RBFs-LSM without polynomial term defined in Eq. (5), because we are not coupling polynomials with LRBFs. The evolution history of the computational results is depicted in Fig. 3. It is evident from the comparison of optimization histories of the methods the LRBFs-LSM the GRBFs-LSM that the optimization process converged in the same numbers of iterations resulting in similar optimal designs. However, the evolution history of the evolving structures shows slight variations from each other for the same number of iterations. The compliance and volume convergence histories alongwith final compliances of LRBFs and GRBFs are shown in Figs. $4 \mathrm{a}$ and $4 \mathrm{~b}$, respectively. 
Table 2: CPU time comparison in term of seconds of GRBFs, CS-RBFs and LRBFs of test problem 1.

\begin{tabular}{ccccccc}
\hline \multirow{2}{*}{$\mathrm{N}$} & CPU Time & \multicolumn{5}{c}{ Iterations } \\
\cline { 2 - 7 } & GRBFs & CS-RBFs & LRBFs & GRBFs & CS-RBFs & LRBFs \\
\hline $40 \times 20$ & 13.48 & 13.10 & 13.22 & 159 & 146 & 132 \\
$60 \times 30$ & 16.21 & 20.05 & 16.56 & 96 & 116 & 96 \\
$80 \times 40$ & 43.63 & 43.36 & 40.16 & 139 & 137 & 139 \\
$100 \times 50$ & 91.09 & 148.86 & 53.52 & 163 & 286 & 103 \\
$120 \times 60$ & 150.79 & 139.76 & 96.80 & 154 & 150 & 114 \\
\hline
\end{tabular}

In order to further validate and explore the possible advantages/ shortcomings of the proposed approach in terms of sensitivity to shape parameter selection, computational efficiency, mesh dependency, etc, some numerical experiments are carried out for the problem shown in Fig. 2a, using GRBFs, CS-RBFs and LRBFs, respectively. To the authors knowledge this investigation have never been carried out previously. Hence, the main objective of this present implementation can be considered as a step forward for further enhancement of the use of RBFs for the solution of classical structural shape and topology optimization problems within the LSM framework, initially proposed in $[35,38]$.

In our numerical experiments we have observed that the GRBFs-LSM is sensitive to the selection of the shape parameter. This method gives the optimal solution for $c \in(0,7]$. However, For $c \geq 7$ the GRBFs moment matrix become highly ill-conditioned without obtaining the optimum solution. Also the CS-RBFs-LSM gives the optimal solution for values of $c$ in the interval $c \in(0,1.7]$, where as for $c \geq 1.8$ the moment matrix of CS-RBFs becomes seriously ill-conditioned. On the other hand, the proposed method i.e. LRBFs-LSM provides optimal solutions for any positive integer value of $c \in(1,200)$, as depicted for $c=15,35,100,130,150,180$, respectively in Fig. 5.

Different mesh sizes of $40 \times 20,60 \times 30,80 \times 40,100 \times 50$, and $120 \times 60$ are used, respectively, for the mesh dependency study. The optimal topologies obtained for each of the mesh size are shown in Fig. 6. It can be seen that the LRBFs-LSM produces almost the same optimal topologies with little variations in comparison with global and compactly supported RBFs. The less mesh independence of the LRBFs-LSM is obtained due to variation of the stencil size, which is not possible in the GRBFs-LSM. This suggests that this new implementation is a step forward towards further improvements of RBFs in the LSM framework.

Furthermore, a comparison of the CPU time (sec) has also been carried out to evaluate the computational efficiency using different mesh sizes. Since with the LRBFs-LSM their is no need to calculate the inverse of $H$ matrix in time loop, we have calculated the inverse of $H$ matrix in Eq. (20) in GRBFs and CS-RBFs based LSM outside the time loop, rather than inside the loop as in [58]. Results obtained are presented in Table 2, which suggests computationally efficiency 


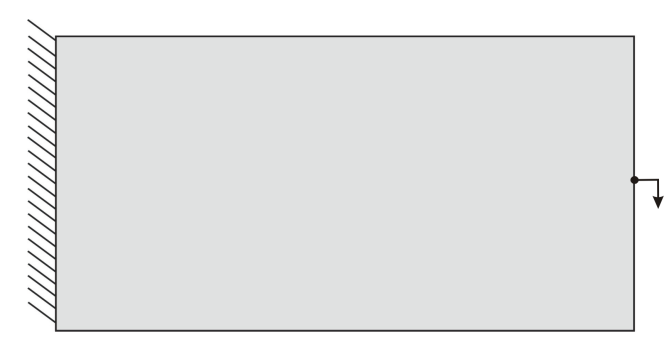

(a) Initial design

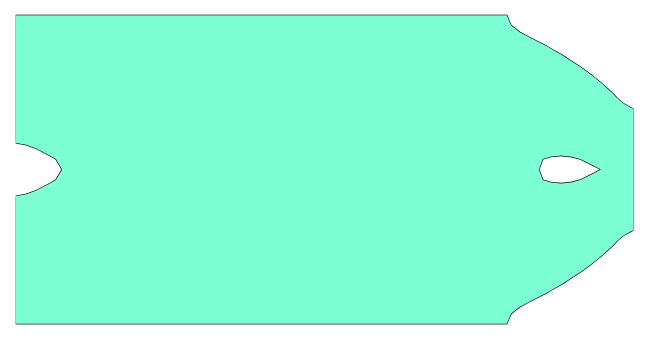

(c) Step 30

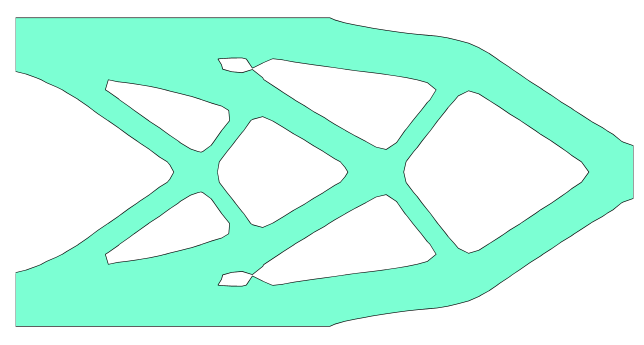

(e) Step 60

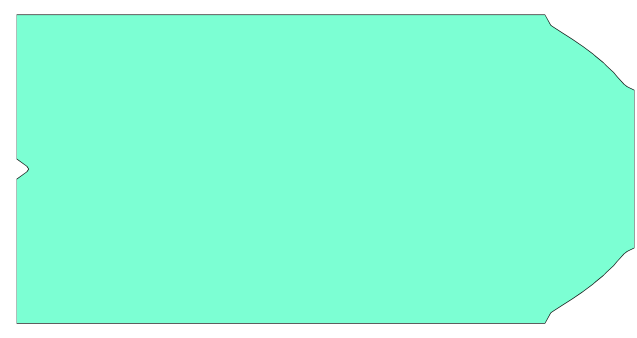

(b) Step 20

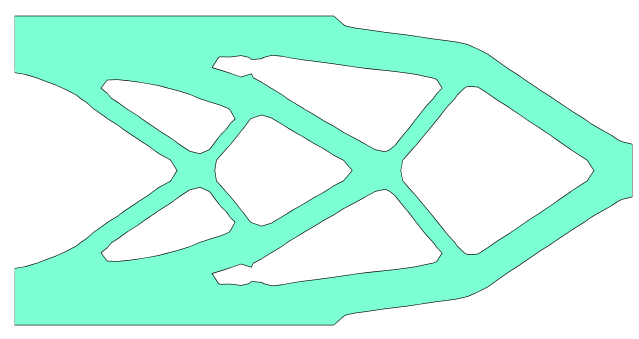

(d) Step 50

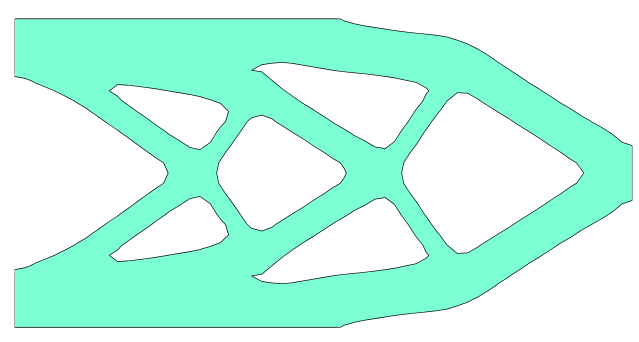

(f) Optimal solution

Figure 2: Evolution of structural geometry using the LRBFs-LSM, for problem 1. 

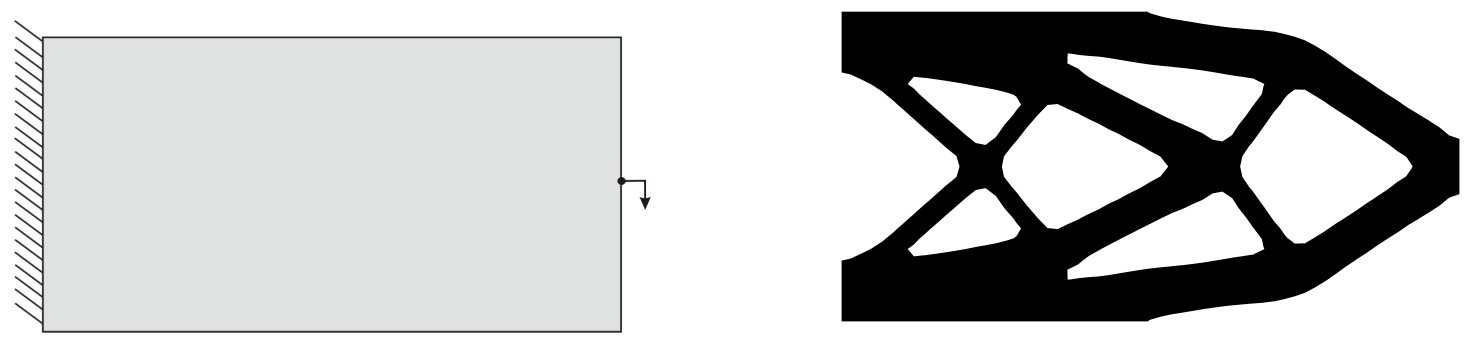

(a) Initial design

(b) Optimal solution

Figure 3: Evolution of structural geometry using the GRBFs-LSM, for problem 1

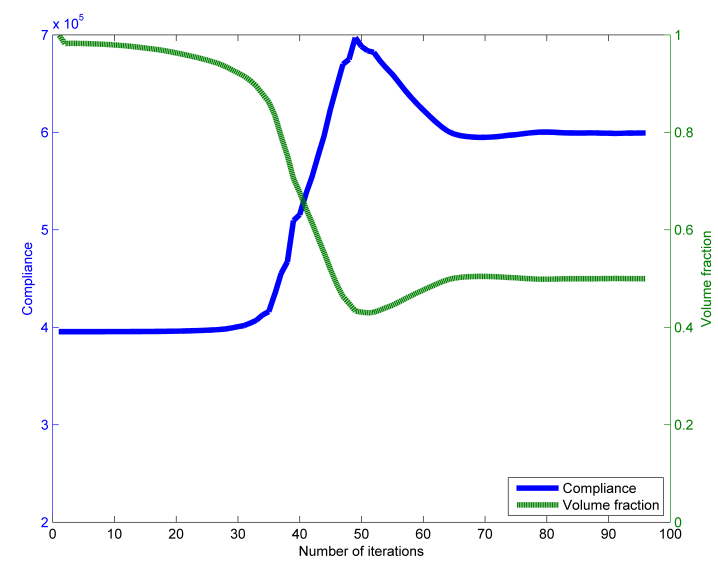

(a) LRBFs-LSM

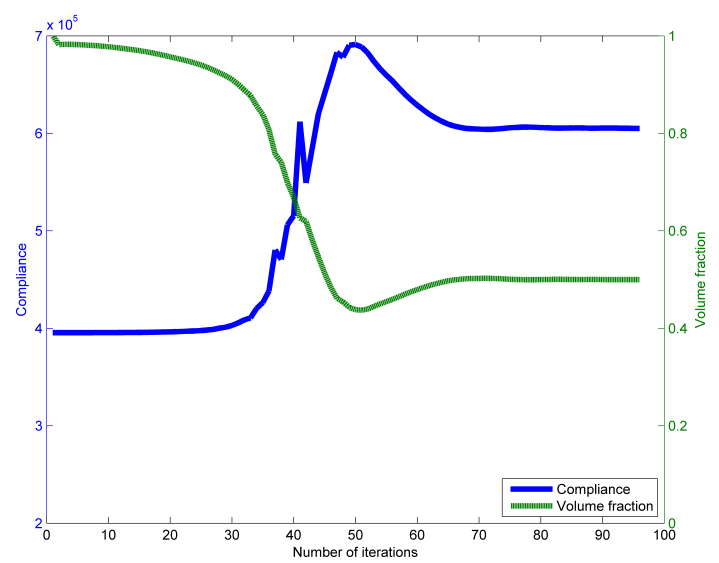

(b) GRBFs-LSM in [58]

Figure 4: Objective function and volume convergence for test problem 1. 

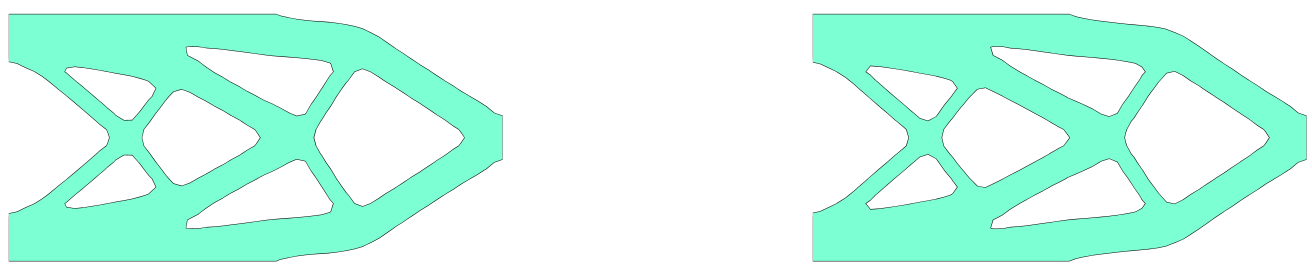

(a) $c=15$

(b) $c=35$
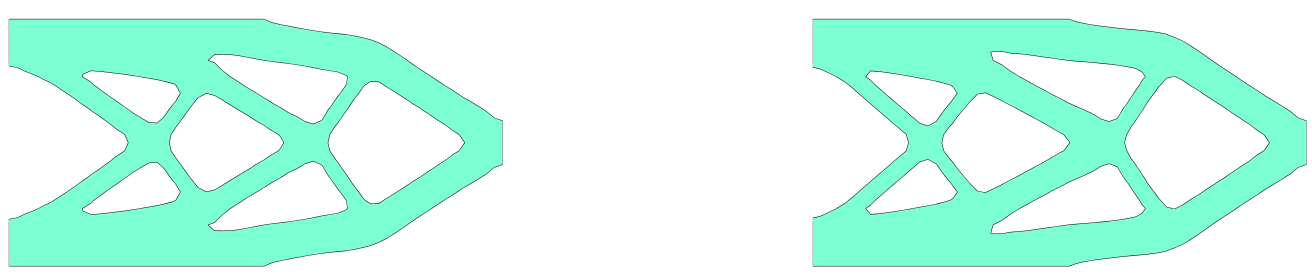

(c) $c=100$

(d) $c=130$
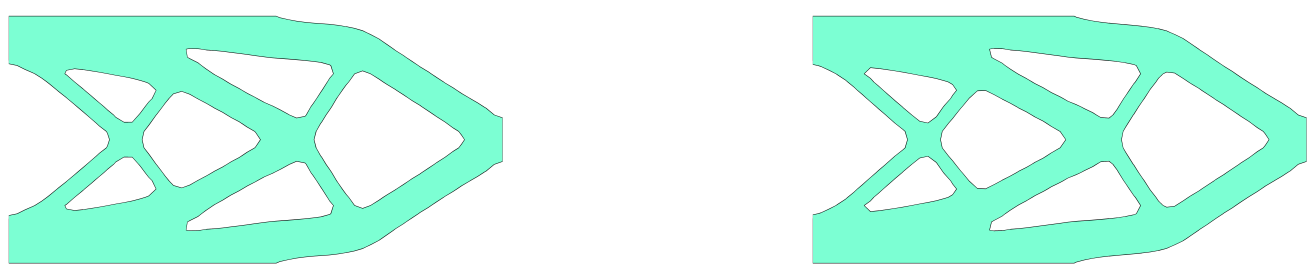

(e) $c=150$

(f) $c=180$

Figure 5: Optimal topologies for different values of shape parameter $c$, for problem 1 . 


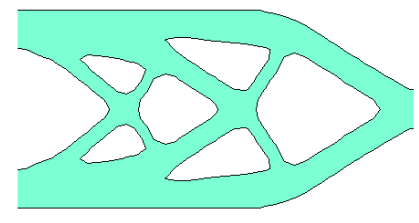

(a) $40 \times 20$

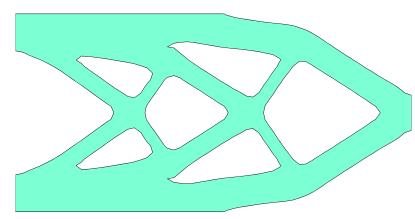

(d) $60 \times 30$

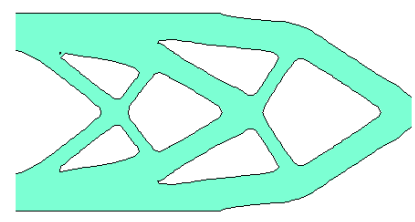

(g) $80 \times 40$

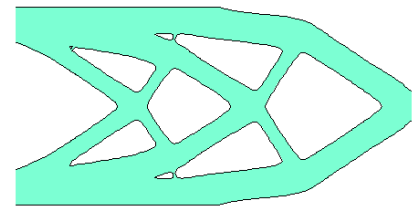

(j) $100 \times 50$

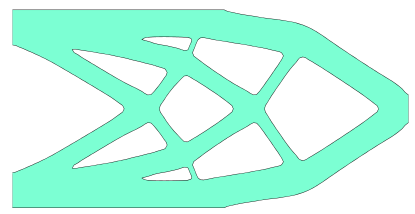

(m) $120 \times 60$

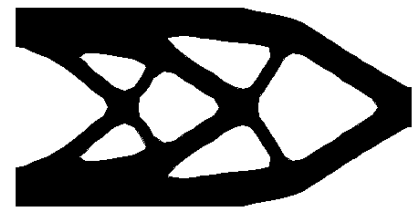

(b) $40 \times 20$

(c) $40 \times 20$
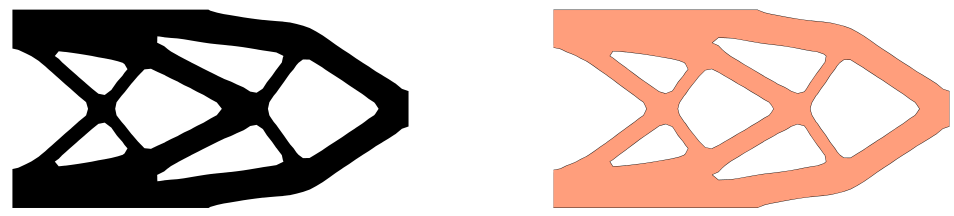

(e) $60 \times 30$

(f) $60 \times 30$
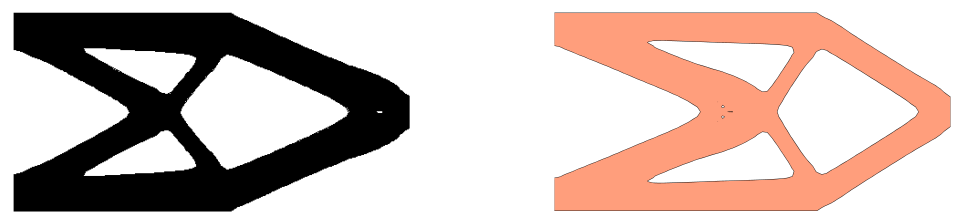

(h) $80 \times 40$

(i) $80 \times 40$
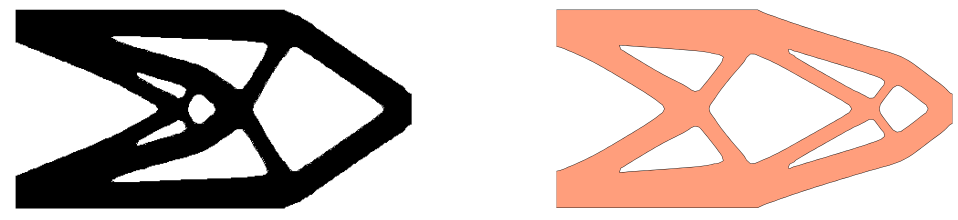

(k) $100 \times 50$

(l) $100 \times 50$

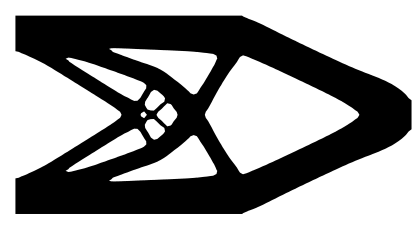

(n) $120 \times 60$

(o) $120 \times 60$

Figure 6: Mesh dependency study, for problem 1 using the methods LRBFs-LSM first column, GRBFs-LSM second column and CS-RFBs-LSM5third column (from left to right). 
of the present approach.

Test Problem 2. Consider a cantilever beam with an aspect ratio of $1: 1$, with a vertical force at the right bottom edge corner. The beam is fixed at the top and bottom of the left edge. The range of values of the shape parameter is taken $[1,100]$ to obtain the optimized structure. A mesh of size $30 \times 30=900$ is used in this problem, with $c=2$.

The initial structure with the applied load and boundary conditions is given in Fig. 7a. The required volume fraction is $45 \%$ for this problem. Figs. $7 \mathrm{~b}-7$ e show the evolution history, where the final optimal structure is obtained after 160 iteration. The optimal design with the LRBFs and LSM is very similar to that reported in the literature e.g $[10,22,66]$.

Further validation of the proposed method for this test problem is also carried out as depicted in Fig. 8. The compliance and volume histories of both the methods are illustrated in Fig. 9, showing a smooth convergent behaviour of the proposed method.

Test Problem 3. Next we apply the present method to Michell type structure having an aspect ratio of $2: 1$. The structure has zero displacement in all directions at the right and left hand sides of the bottom edge and a vertical force is applied at the centre of the bottom edge. The value of shape parameter $c=20$. The LRBFs-LSM gives optimal solutions for $c \in[1,100]$ for the initial structure shown in Fig. 10a. A mesh size of $60 \times 30=1800$ is used for this problem.

The required volume fraction is $50 \%$. The evolution history of structural configuration at different iterations is given in Fig. 10, which is very similar to the optimal designs in the literature $[38,67]$. The hole insertion is evident throughout the optimization process. This test case shows that the proposed method has the capability for the solution of different types of structural topology optimization problems. The convergence histories of the compliance and volume fraction is depicted in Fig. 11, which shows a smoother evolution of both the factors during the optimization process.

Test Problem 4. Finally, we consider the multiple load case as shown in Fig. 12a. The beam is constrained at the right and left corners of the bottom edge. Three loads, one at the center of the bottom boundary in downward direction and the other two loads in opposite horizontal directions at the point preceded from the center are applied. The shape parameter used in this problem is 5 . A mesh size of $60 \times 30=1800$ is used to discretize the structural domain.

The initial topology of the structure along with boundary and loading conditions is shown in Fig. 12a. The required volume fraction for this problem is set to 50\%. Fig. 12 shows the complete geometry evolution history where the final optimal topology is obtained after 116 iterations as shown $\mathrm{n}$ Fig. 12f. This test problem further verifies versatility and capability of he proposed method in multiple load cases as well. 


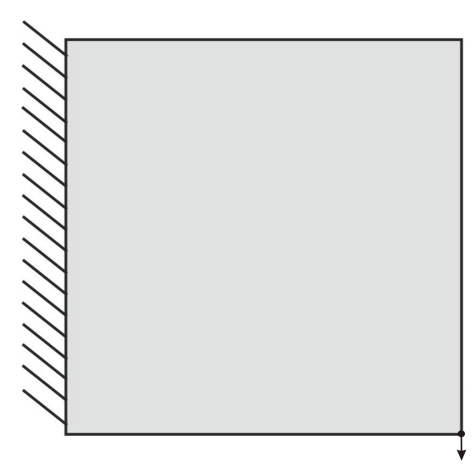

(a) Initial design

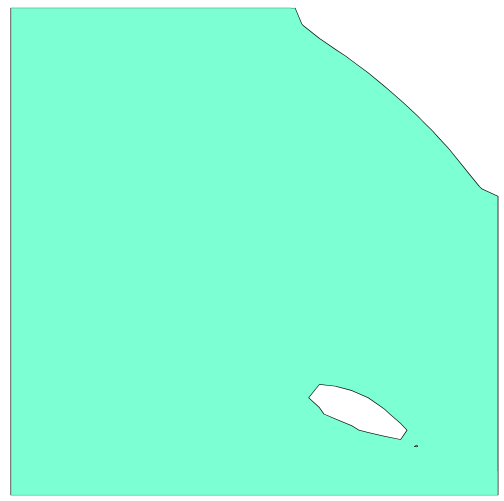

(c) Step 20

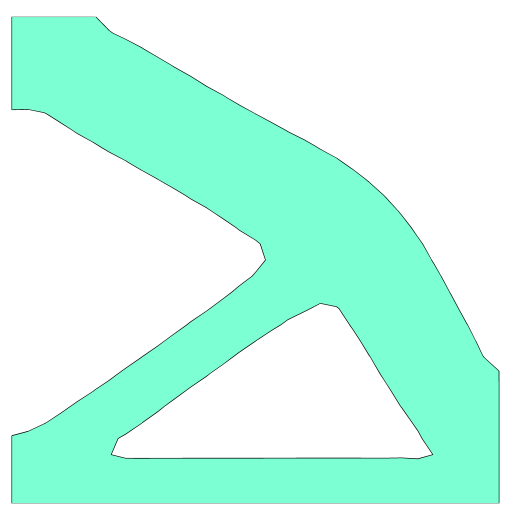

(e) Step 40

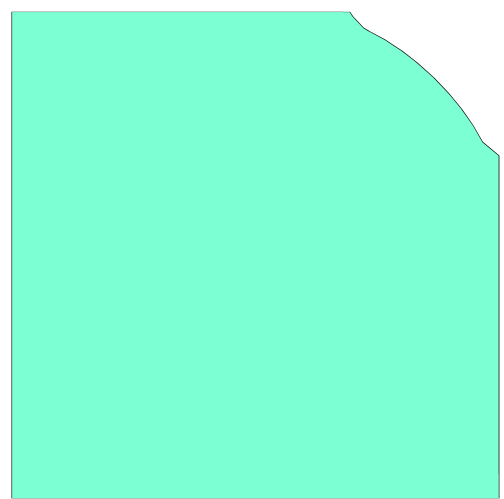

(b) Step 10

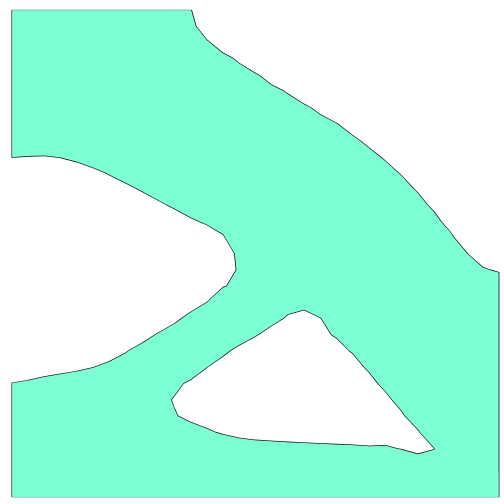

(d) Step 30

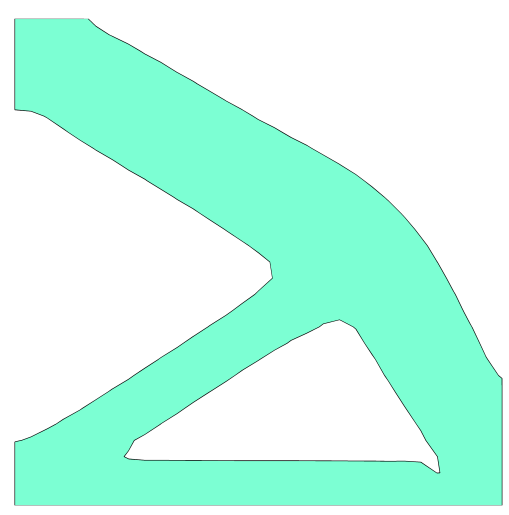

(f) Optimal solution

Figure 7: Evolution of structural geometry using LRBFs-LSM, for problem 2. 


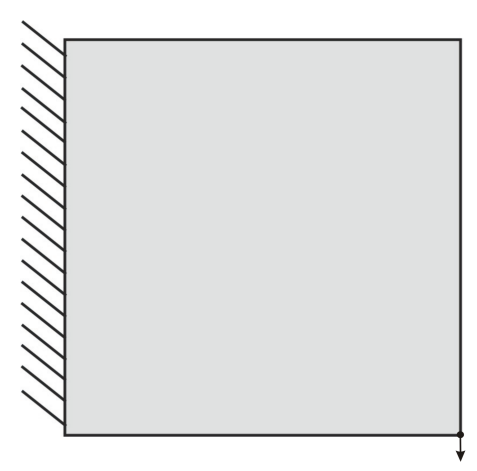

(a) Initial design

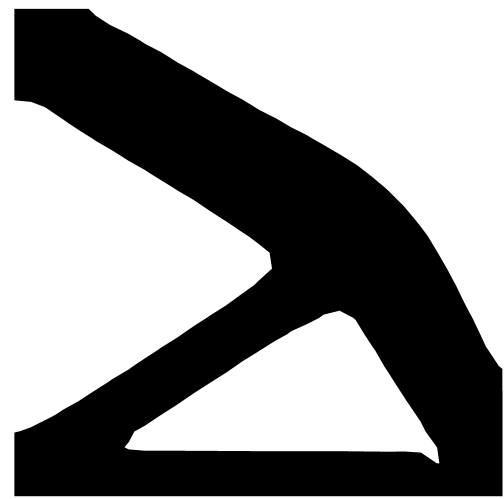

(b) Final solution

Figure 8: Optimal solution of test problem 2 using GRBFs-LSM.

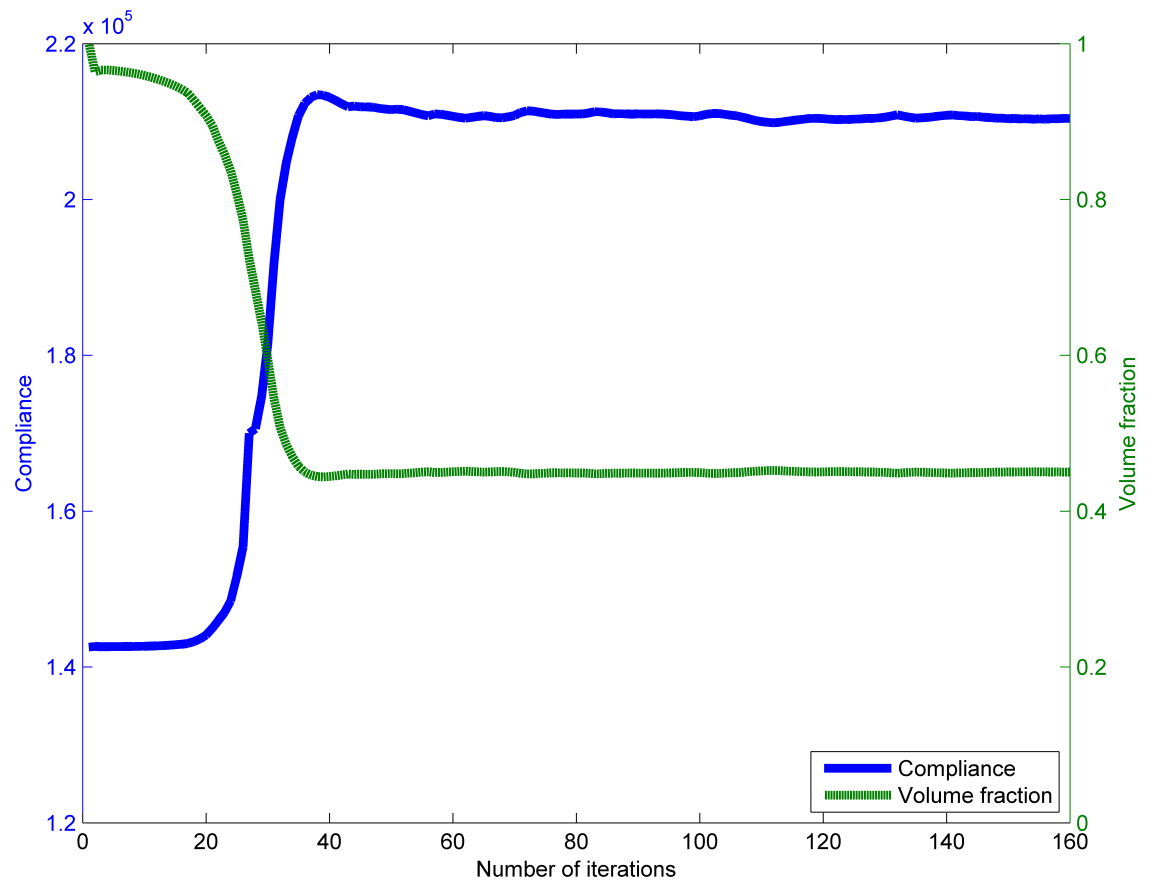

Figure 9: Objective function and volume convergence for test problem 2. 


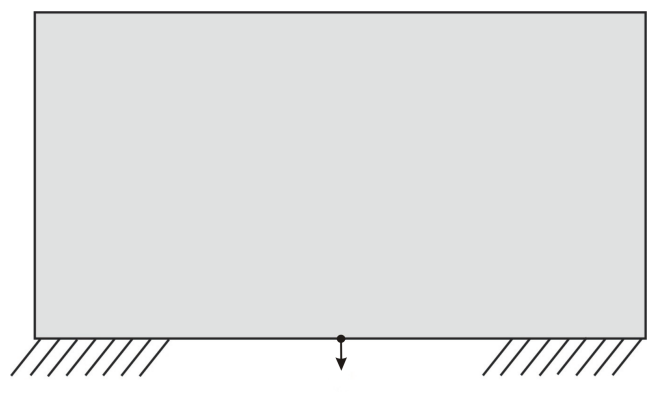

(a) Initial design

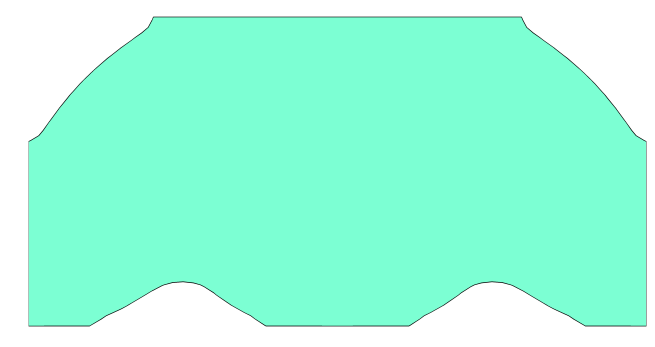

(c) Step 20

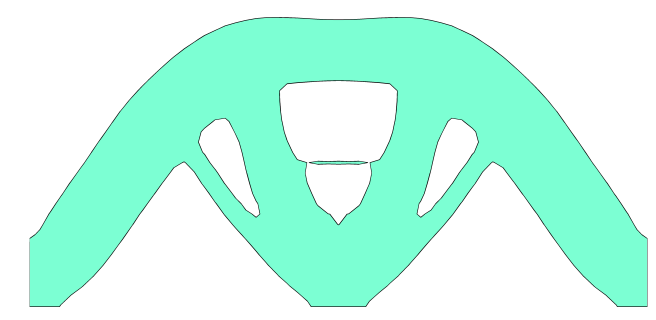

(e) Step 40

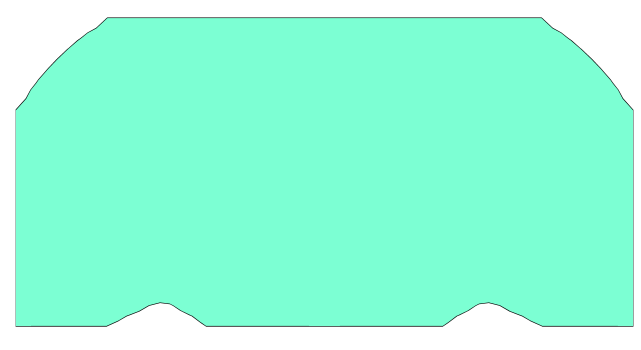

(b) Step 10

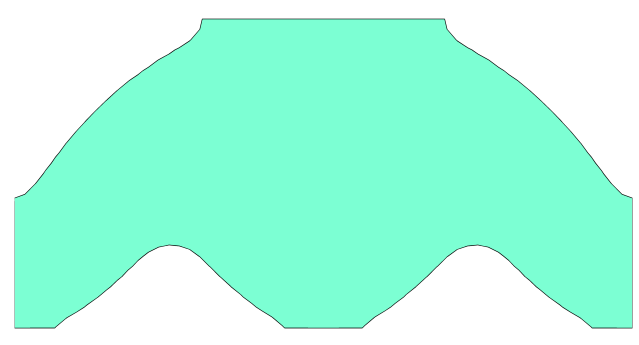

(d) Step 30

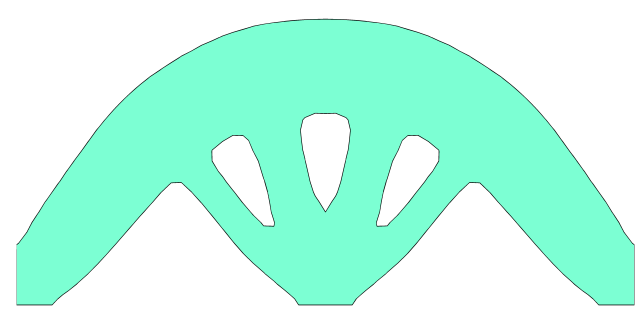

(f) Optimal solution

Figure 10: Evolution of structural geometry using LRBFs-LSM, for problem 3. 


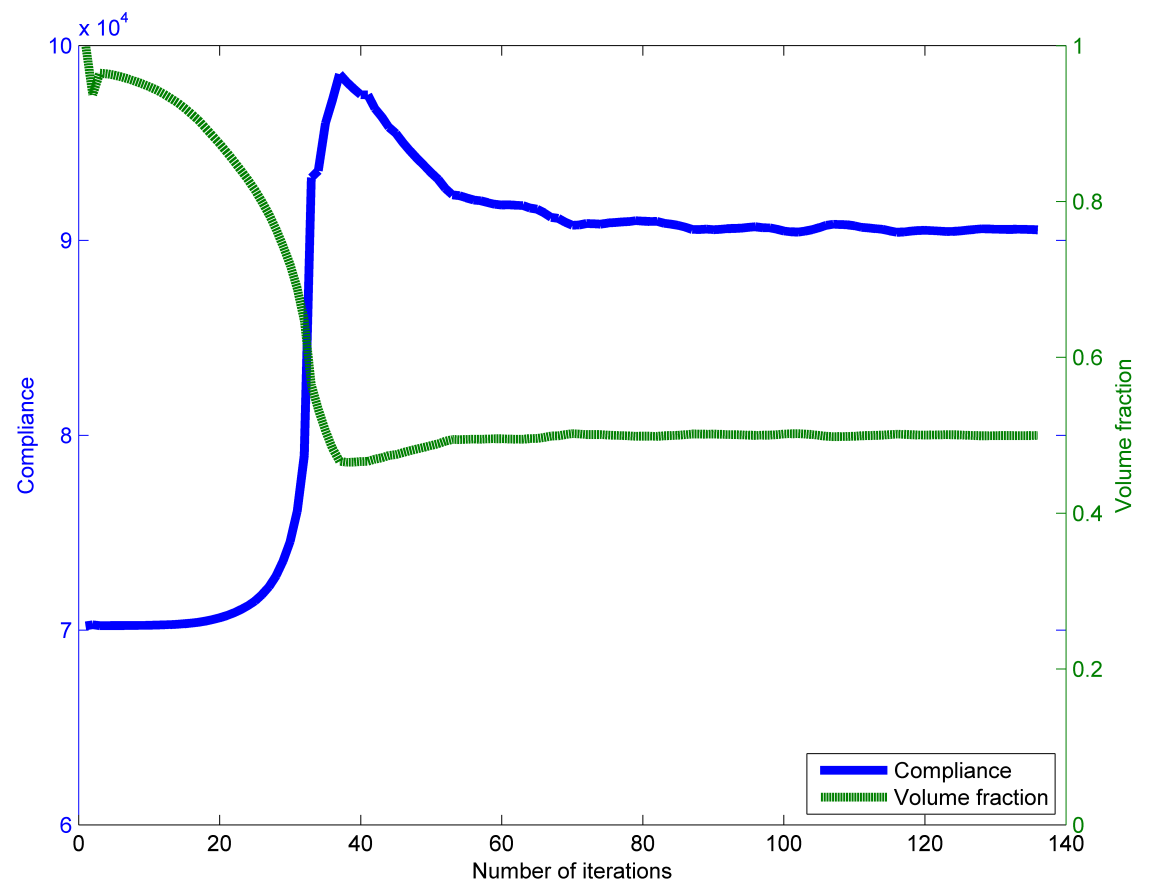

Figure 11: Objective function and volume convergence for test problem 3. 


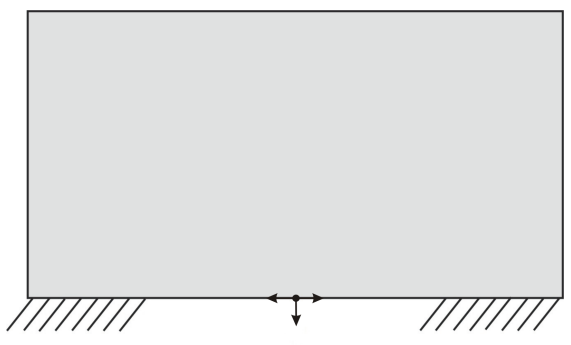

(a) Initial design

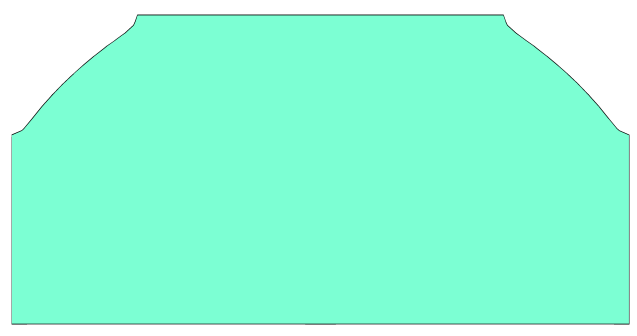

(c) Step 20

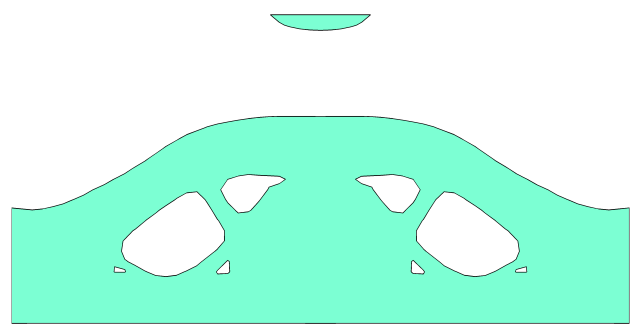

(e) Step 40

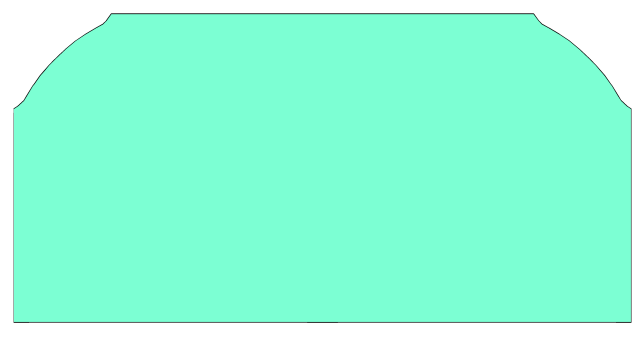

(b) Step 10

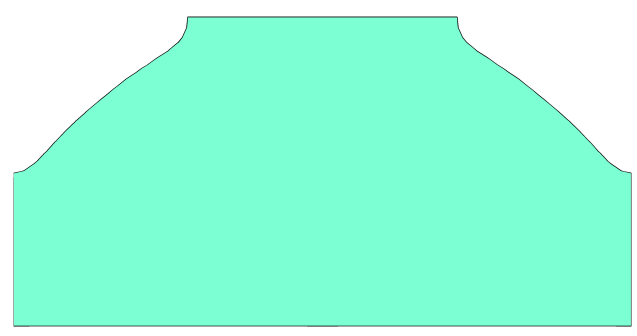

(d) Step 30

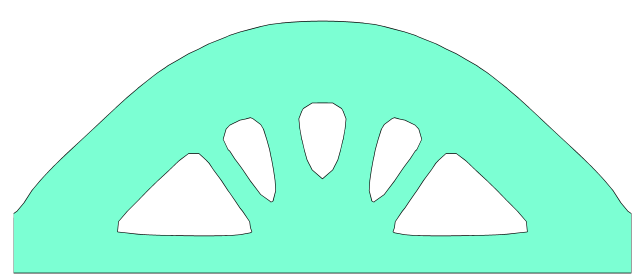

(f) Optimal solution

Figure 12: Evolution of structural geometry using LRBFs-LSM, for problem 4. 


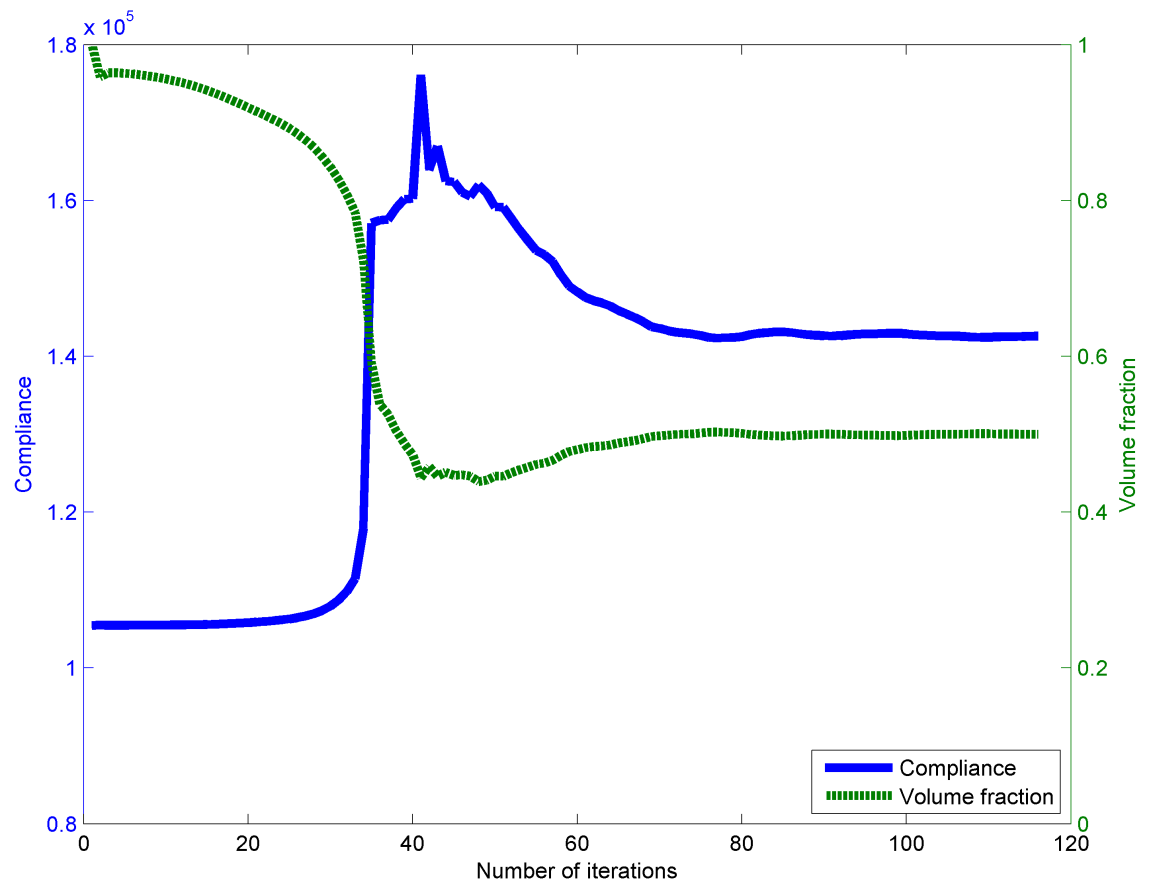

Figure 13: Objective function and volume convergence for problem 4. 


\section{Conclusions}

In this paper a localized version of the multi-quadric (MQ) radial basis functions based level set method is proposed for the two dimensional structural optimization problems. The nature of the wok performed aims to highlight the advantages and possible improvements in the context of structural optimization algorithms.

- The propagation of the level set function is tracked through a solution of ODEs rather than a PDEs by the LSM based on LRBFs.

- Hole nucleation at the appropriate location having minimum strain energy within the design domain is handled automatically by the proposed implementation. Thereby, avoiding the dependency of the final optimal solution on the initial design domain.

- The computational efficiency of the LRBFs-LSM is established over the methods reported in the literature of structural optimization.

- The localized RBFs are less sensitive to the selection of shape parameter values.

- Numerical tests confirm that the localized version of RBFs is mesh independent.

- Numerical test problems having a single and multiple load applications confirm the use of the present method as a step forward for further exploration of the applicability of RBFs in LSM and topology optimization framework.

\section{Acknowledgment}

The authors acknowledge financial assistance from the Higher Education Commission Islamabad Pakistan through NRPU Project No. 6331.

\section{References}

[1] G. I. Rozvany, A critical review of established methods of structural topology optimization, Structural and Multidisciplinary Optimization 37 (3) (2009) 217-237.

[2] M. P. Bendsoe, N. Kikuchi, Generating optimal topologies in structural design using a homogenization method, Computer Methods in Applied Mechanics and Engineering 71 (1988) $197-224$.

[3] K. Suzuki, N. Kikuchi, A homogenization method for shape and topology optimization, Computer Methods in Applied Mechanics and Engineering 93 (3) (1991) 291-318.

[4] G. Allaire, R. V. Kohn, Optimal design for minimum weight and compliance in plane stress using extremal microstructures, European Journal of Mechanics. A. Solids 12 (6) (1993) 839-878.

[5] O. Sigmund, J. Petersson, Numerical instabilities in topology optimization: a survey on procedures dealing with checkerboards, mesh-dependencies and local minima, Structural Optimization 16 (1) (1998) 68-75. 
[6] S. Osher, J.A. Sethian, Front propagating with curvature dependent speed: algorithms based on HamiltonJacobi formulations, Journal of Computational Physics 78 (1988) 12-49.

[7] J. A. Sethian, Level set methods and fast marching methods: evolving interfaces in computational geometry, fluid mechanics, computer vision and materials science., Vol. 3, Cambridge Monographs on Applied and Computational Mathematics, Cambridge University Press, Cambridge, 1999.

[8] J. A. Sethian, A. Wiegmann, Structural boundary design via level set and immersed interface methods, Journal of Computational Physics 163 (2) (2000) 489-528.

[9] J. C. Ye, Y. Bresler, P. Moulin, A self-referencing level-set method for image reconstruction from sparse fourier samples, International Journal of Computer Vision 50 (3) (2002) 253-270.

[10] M. Y. Wang, X. Wang, D. Guo, A level set method for structural topology optimization, Computer methods in applied mechanics and engineering 192 (1-2) (2003) 227-246.

[11] G. Allaire, F. Jouve, A.-M. Toader, Structural optimization using sensitivity analysis and a level-set method, Journal of Computational Physics 194 (1) (2004) 363-393.

[12] Osher S, Fedkiw RP, Level set methods: an overview and some recent results, Journal of Computational Physics 169 (2) (2001) 463-502.

[13] F. Osher S, Level set methods and dynamic implicit surfaces, Springer, New York.

[14] H. Ghasemi, H. S. Park, T. Rabczuk, A multi-material level set-based topology optimization of flexoelectric composites, Computer Methods in Applied Mechanics and Engineering 332 (2018) 47-62.

[15] S. Yamasaki, T. Yamada, T. Matsumoto, An immersed boundary element method for levelset based topology optimization, International Journal for Numerical Methods in Engineering 93 (9) (2013) 960-988.

[16] H. Isakari, K. Kuriyama, S. Harada, T. Yamada, T. Takahashi, T. Matsumoto, A topology optimisation for three-dimensional acoustics with the level set method and the fast multipole boundary element method, Mechanical Engineering Journal 1 (4) (2014) 1-13.

[17] P. C. Vitório, E. D. Leonel, Topology optimization analysis based on the direct coupling of the boundary element method and the level set method, International Journal of Advanced Structural Engineering 9 (4) (2017) 397-407.

[18] T. Yamada, K. Izui, S. Nishiwaki, A. Takezawa, A topology optimization method based on the level set method incorporating a fictitious interface energy, Computer Methods in Applied Mechanics and Engineering 199 (45-48) (2010) 2876-2891.

[19] Y. Noguchi, T. Yamada, K. Izui, S. Nishiwaki, Topology optimization for hyperbolic acoustic metamaterials using a high-frequency homogenization method, Computer Methods in Applied Mechanics and Engineering https://doi.org/10.1016/j.cma.2018.02.031.

[20] T. Yamada, K. Izui, S. Nishiwaki, A level set-based topology optimization method for maximizing thermal diffusivity in problems including design-dependent effects, Journal of Mechanical Design 133 (3) (2011) 031011-1. 
[21] Z. Luo, N. Zhang, W. Gao, H. Ma, Structural shape and topology optimization using a meshless Galerkin level set method, International Journal for Numerical Methods in Engineering 90 (3) (2012) 369-389.

[22] W. Khan, Siraj-ul-Islam, B. Ullah, Structural optimization based on meshless element free Galerkin and level set methods, Computer Methods in Applied Mechanics and Engineering 344 (2019) 144-163.

[23] S. Osher, R. P. Fedkiw, Level set methods: an overview and some recent results, Journal of Computational Physics 169 (2) (2001) 463-502.

[24] N. P. Van Dijk, K. Maute, M. Langelaar, F. Van Keulen, Level-set methods for structural topology optimization: a review, Structural and Multidisciplinary Optimization 48 (3) (2013) $437-472$.

[25] A. L. Gain, G. H. Paulino, A critical comparative assessment of differential equation-driven methods for structural topology optimization, Structural and Multidisciplinary Optimization 48 (4) (2013) 685-710.

[26] Chopp, D. Layne, Computing minimal surfaces via level set curvature flow, Ph.D. thesis, University of California, Berkeley, CA 94720 (1991).

[27] S. Yamasaki, S. Nishiwaki, T. Yamada, K. Izui, M. Yoshimura, A structural optimization method based on the level set method using a new geometry-based re-initialization scheme, International Journal for Numerical Methods in Engineering 83 (12) (2010) 1580-1624.

[28] M. Sussman, P. Smereka, S. Osher, A level set approach for computing solutions to incompressible two-phase flow, Journal of Computational Physics 114 (1) (1994) 146-159.

[29] J. Sokolowski, J.-P. Zolesio, Introduction to shape optimization, Springer, 1992.

[30] G. Allaire, F. De Gournay, F. Jouve, A.-M. Toader, Structural optimization using topological and shape sensitivity via a level set method, Control and cybernetics 34 (1) (2005) 59.

[31] M. Y. Wang, P. Wei, Topology optimization with level set method incorporating topological derivative, 6th World Congress on Structural and Multidisciplinary Optimization (2005) $761-769$.

[32] M. Burger, B. Hackl, W. Ring, Incorporating topological derivatives into level set methods, Journal of Computational Physics 194 (1) (2004) 344-362.

[33] V. J. Challis, A discrete level-set topology optimization code written in matlab, Structural Multidisciplinary Optimization 41 (2010) 453-464.

[34] M. Burger, Lecture notes on infinite-dimensional optimization and optimal design, Report 285J, University of California, Los Angeles, CA, USA (2004).

[35] S. Wang, M. Y. Wang, Radial basis functions and level set method for structural topology optimization, International Journal for Numerical Methods in Engineering 65 (12) (2006) 2060-2090.

[36] M. D. Buhmann, Radial basis functions: theory and implementations, Vol. 12, Cambridge University press, 2003. 
[37] B. Šarler, R. Vertnik, Meshfree explicit local radial basis function collocation method for diffusion problems, Computers \& Mathematics with applications 51 (8) (2006) 1269-1282.

[38] S. Wang, K. Lim, B. C. Khoo, M. Y. Wang, An extended level set method for shape and topology optimization, Journal of Computational Physics 221 (1) (2007) 395-421.

[39] Y. Liu, Z. Li, P. Wei, W. Wang, Parameterized level-set topology optimization method considering symmetry and pattern repetition constraints, Computer Methods in Applied Mechanics and Engineering 340 (2018) 1079-1101.

[40] Ho, Hon Shan, Wang, Michael Yu, Zhou, Mingdong, Parametric structural optimization with dynamic knot RBFs and partition of unity method, Structural and Multidisciplinary Optimization 47 (3) (2013) 353-365.

[41] X. Xie, M. Mirmehdi, Radial basis function based level set interpolation and evolution for deformable modelling, Image and Vision Computing 29 (2-3) (2011) 167-177.

[42] Siraj-ul-Islam, R. Vertnik, B. Šarler, Local radial basis function collocation method along with explicit time stepping for hyperbolic partial differential equations, Applied Numerical Mathematics 67 (2013) 136-151.

[43] Siraj-ul-Isalm, I. Ahmad, A comparative analysis of local meshless formulation for multiasset option models, Engineering Analysis with Boundary Element 65 (2016) 159-176.

[44] Z. Luo, L. Tong, M. Y. Wang, S. Wang, Shape and topology optimization of compliant mechanisms using a parameterization level set method, Journal of Computational Physics 227 (1) (2007) 680-705.

[45] Z. Luo, M. Y. Wang, S. Wang, P. Wei, A level set-based parameterization method for structural shape and topology optimization, International Journal for Numerical Methods in Engineering 76 (1) (2008) 1-26.

[46] Z. Luo, L. Tong, A level set method for shape and topology optimization of largedisplacement compliant mechanisms, International Journal for Numerical Methods in Engineering 76 (6) (2008) 862-892.

[47] Z. Luo, L. Tong, Z. Kang, A level set method for structural shape and topology optimization using radial basis functions, Computers \& Structures 87 (7-8) (2009) 425-434.

[48] R. Schaback, H. Wendland, Using compactly supported radial basis functions to solve partial differential equations, WIT Transactions on Modelling and Simulation 23 (1970).

[49] C. Chen, M. Golberg, R. Schaback, Recent developments in the dual reciprocity method using compactly supported radial basis functions, Transformation of Domain Effects to the Boundary 1 (2003) 1-41.

[50] G. Yao, J. Duo, C. Chen, L. Shen, Implicit local radial basis function interpolations based on function values, Applied Mathematics and Computation 265 (2015) 91-102.

[51] N. Flyer, G. B. Wright, B. Fornberg, Radial basis function-generated finite differences: A mesh-free method for computational geosciences, Handbook of Geomathematics (2014) 1-30. 
[52] J.-S. Chen, L. Wang, H.-Y. Hu, S.-W. Chi, Subdomain radial basis collocation method for heterogeneous media, International Journal for Numerical Methods in Engineering 80 (2) (2009) 163-190.

[53] G. Kosec, B. Sarler, Simulation of macrosegregation with mesosegregates in binary metallic casts by a meshless method, Engineering Analysis with Boundary Elements 45 (2014) 36-44.

[54] R. Vertnik, B. Sarler, Solution of incompressible turbulent flow by a mesh-free method, Computer Modeling in Engineering and Sciences (CMES) 44 (1) (2009) 65.

[55] I. Ahmad, et al., A comparative analysis of local meshless formulation for multi-asset option models, Engineering Analysis with Boundary Elements 65 (2016) 159-176.

[56] B. B. Kee, G. Liu, C. Lu, A least-square radial point collocation method for adaptive analysis in linear elasticity, Engineering Analysis with Boundary Elements 32 (6) (2008) 440-460.

[57] W. Khan, Siraj-ul-Islam, B. Ullah, Analysis of meshless weak and strong formulations for boundary value problems, Engineering Analysis with Boundary Elements 80 (2017) 1-17.

[58] P. Wei, Z. Li, X. Li, M. Y. Wang, An 88-line matlab code for the parameterized level set method based topology optimization using radial basis functions, Structural and Multidisciplinary Optimization (2018) 1-19.

[59] S. Osher, J. A. Sethian, Fronts propagating with curvature-dependent speed: algorithms based on hamilton-jacobi formulations, Journal of Computational Physics 79 (1) (1988) 12 49 .

[60] R. Tsai, S. Osher, Level set methods and their applications in image science, Communications in Mathematical Sciences 4 (1) (2003) 623-656.

[61] E. Kansa, H. Power, G. Fasshauer, L. Ling, A volumetric integral radial basis function method for time-dependent partial differential equations. I. formulation, Engineering Analysis with Boundary Elements 28 (10) (2004) 1191-1206.

[62] R. Franke, Scattered data interpolation: tests of some methods, Mathematics of Computation 38 (157) (1982) 181-200.

[63] M. E. Chenoweth, A local radial basis function method for the numerical solution of partial differential equations, Master's thesis, Marshall University (2012).

[64] B. Ullah, J. Trevelyan, A boundary element and level set based topology optimisation using sensitivity analysis, Engineering Analysis with Boundary Elements 70 (2016) 80-98.

[65] H. A. Jahangiry, S. M. Tavakkoli, An isogeometrical approach to structural level set topology optimization, Computer Methods in Applied Mechanics and Engineering 319 (2017) 240-257.

[66] B. Ullah, J. Trevelyan, P.C. Matthews, Structural optimisation based on the boundary element and level set methods, Computers and Structures 137 (2014) 14-30.

[67] B. Ullah, Structural topology optimisation based on the boundary element and level set methods, Ph.D. thesis, Durham University (2014). 